Some will be inclined to ask how such serum exerts its protective properties. I cannot answer the question. Certainly I think it is not a chemical antidote, but it is an established fact that susceptible animals adequately protected so as to reach the highest possible degree of immunity from infection with pathogenic bacteria or from intoxications with their chemical products are capable of supplying a serum which injected in to other susceptible animals will protect them against like infections and intoxications, and also in some cases have a curative effect. How it is capable of exerting this power seems to be unknown. Various hypotheses have been advanced, and I cannot do better than quote from an article by Dr Kanthack published only last year. He says, "the mechanism of immunity, whetheracquïred or natural, specific or general, is still hidden from us, and we cannot as yet lift the veil, yet the recent past has revealed so many marvellous facts unto us that we may confidently look to the future for more light" (Allbutt).

Before concluding let me point out that there is no universal method of standardising the strength of serum. We are compelled to accept the word of the manufacturer as to its strength and the dose we should administer. Unfortunately a commercial spirit seerns to have pervaded some of the vendors, who advertise in a puffy way worthy of cocoa or soap. It behoves us not to pay too much attention to this, but, bearing in mind that the serum, from the cost involved in its preparation, must be very expensive, buy only from well-known sources.

\title{
SOME FATAL CASES OF PNEUMONIA AND PLEURISY IN THE HORSE*
}

\author{
By Veterinary-Major F. Smith, Army Veterinary Department.
}

THE fatal cases here recorded are those which have occurred in my practice during the last two and a half years; they form the basis of the article on pneumonia and pleurisy published in this Journal (vol. $X .$, Nos. I and 2 ), and the paper in question may perhaps be consulted with advantage when, from the necessarily brief manner in which the clinical records are reported, any point in connection with the chest sounds may appear obscure. All the horses were young, the majority being four years old.

I have drawn certain conclusions from the recorded facts; those having a particular bearing on any special case are inserted under the head of "Remarks." The other conclusions arrived at have appeared in the paper above mentioned.

The value of the present record lies in the fact that it is complete from invasion to death; and, secondly, the facts being correct, the reader is placed in a position to make his own deductions, and is not bound down by those of the observer.

\section{PNEUMONIA.}

Case $I$.

Short History. Admitted with symptoms of fever, but no lung trouble discovered. On the second day the right lung became 
affected. On the third day the symptoms became very urgent, great depression, grunting, and respiratory distress ; hardly any air appeared to enter the left lung, and very little entered the right. On the fifth day very great distress, no respiratory murmur can be detected in either lung, but the chest perfectly resonant from top to bottom; pulse fair. On the sixth day worse; up and down several times during the day owing to distress. Seventh day, died.

Pulse, Respiration, and Teinperature. The pulse started at 70 , for two days remained at 60 , and then rose to 80 . A peculiar pulserespiration ratio was present during the last two days of life, the breathing being 40 to the minute. The temperature rose and fell $2^{\circ}$ every twelve hours for four days.

Post-mortem Appearance. The lungs did not collapse on opening the chest. They were removed with difficulty, but neither cutting nor pressing them caused collapse. The lungs looked mottled in patches all over ; these were isolated patches of pneumonia, and on section they were maroon in colour, spongy, and sharply demarcated from the surrounding healthy tissue. The anterior lobes of both lungs were hepatised, and contained abscesses ; pleura normal.

Remarks. (I) A very remarkable case. I am puzzled to know whether the lungs recoiled to any extent during life, and cannot explain their non-collapse after death. The peculiar pulse-respiration ratio may have been due to this condition of lung. (2) Note the distribution of pneumonia in patches. (3) Observe that though grunting occurred throughout the case there was no pleurisy.

\section{Case II.}

Short History. This horse was admitted with pneumonia of the right lung, followed on the sixth day by pneumonia of the left lung. Throughout the case the chest sounds were indefinite; sometimes the lungs would appear to be clearing, and in a few hours no air sound could be heard. This was repeated two or three times. Throughout the attack the patient grunted persistently; it was quite distressing to hear, but not a trace of pleurisy was found post-mortem. The heart impulse was so violent as to mask the lung sounds in that region. The patient had an excellent appetite. Towards the termination of the case there was great respiratory distress. On the twelfth day an abscess was located in the left lung owing to the squeaking and bubbling which existed. None were diagnosed in the right, though a fairly large one was found post-mortem in a position where it should easily have been found. The patient lived fourteen days.

Outline of Post-mortem Appearances. The lower half of both lungs consolidated ; abscesses plentiful in anterior lobes, and in the posterior part of the middle third of each lung was a relatively large abscess.

\section{Case III.}

Short History. This horse was under treatment for strangles, the throat being much swollen, and the abscesses deep seated and giving rise to difficulty in breathing. I did not like the condition of the lungs on the fourth day of the attack, but the respirations were never more than twelve, and the animal, being intensely nervous, could only be sounded with great difficulty. He could not feed owing to the 
throat trouble, and nutritive enemata did not keep him alive. $\mathrm{He}$ died on the sixteenth day.

Pulse, Respiration and Temperature. The pulse was sixty throughout, the respirations twelve, the temperature generally high, though on the fourth day it touched normal.

Outline of Post-mortem Appearances. Right lung anterior lobe solid and full of abscesses; remaining part of lung normal. Left lung contained a solid patch at the inferior third, and in this was an abscess; the patch was sharply circumscribed by healthy lung tissue. There was no pleurisy. The tissues of the soft palate and pharynx were gangrenous, sloughing, and riddled with abscesses; the latter were also found in the tongue. The guttural pouches were black and contained pus; larynx perfectly healthy.

Remarks. Note that pneumonia was present in spite of twelve respirations per minute. The lung trouble was doubtless due to the strangles organism.

\section{Case IV.}

Short History. This case was remarkable in living thirty-six days with acute lung trouble. The patient had no pneumonia when admitted ; the symptoms set in in thirty-six hours, both lungs being affected. On the sixth day both lungs were blocked below; there was great grunting but no pleurisy. On the eighth day friction was present in the right chest. On the eleventh day there was an improvement, friction had left the right chest, and the left lung was clearer. On the sixteenth day air was entering both lungs to near the bottom, but it was associated with considerable squeaking, and in the left lung with tubular breathing. On the eighteenth day there was a decided relapse, and there were extensive râles in both lungs of a wheezing, bubbling, cooing, and squeaking character. On the twentieth day the patient was worse, there was a harsh respiratory murmur and fine tubular breathing in the left lung, while in the right lung there was marked grunting bronchophony behind the elbow. On the twenty-fifth day there was great muscular wasting in spite of the excellent appetite the patient maintained throughout. At this time both lungs were again dull below on percussion, with an entire absence of air sound. Above the consolidated area there were squeaking crepitations, both fine and coarse. On the twentyninth day the dulness on percussion of both chests had nearly gone, the sound being remarkably resonant. Gradually the case got worse, the breathing. more distressed, constant grunting, great muscular wasting, and death on the thirty-sixth day.

Outline of Post-mortem Examination. Both lungs consolidated below, abscesses in the anterior lobes, and many abscesses scattered throughout the two lungs, especially the upper half; one of these was very large, caused by the union of several smaller ones. Only a trace of the pleuritic patch was found in the right chest, the remainder of the pleura being absolutely normal, though the patient grunted persistently for over a month. The lungs could not be inflated after death owing to a leak.

Remarks. Note (I) That the lungs commenced to break down on the sixteenth day. (2) Between the eleventh and twenty-fourth days there appeared to be a somewhat clearer condition of lung, and 
then the organs once more became consolidated. This has been observed in other cases. (3) The pulse-rate, respiration, and temperature indicated that the condition mentioned in (2) could not be real. (4) The resonant chest a week before death was due to pneumo-thorax. (5) Most abscesses were found where there had been the least pneumonia.

\section{Case V.}

Short History. Admitted with a high temperature, tender throat, and dribbling of saliva from mouth; the chest remained clear for two days, and then pneumonia of the lower third of the right lung developed. The throat became worse, swollen, and indicated the formation of strangles abscess. On the ninth day one burst internally and discharged through the nose. The appetite throughout was bad, and the patient had to be hand fed; the left lung remained clear, and the amount of trouble in the right was never very extensive. The patient died on the thirteenth day. Both pulse-rate and respiration were persistently high during the whole of the attack.

Outline of Post-mortem Examination. The lower third and anterior lobe of right lung consolidated and full of abscesses. Great destruction of the tissues of throat and tongue, deep-seated abscesses in the latter, sloughing and intense thickening of the soft palate, pharynx, and epiglottis; the soft palate was at least I inch thick; the submaxillary glands were full of pus.

Remarks. The pneumonia in this case was probably due to the strangles organism.

\section{Case VI.}

Short History. The peculiar feature in this case was the doubt that existed for some days as to whether pneumonia were present or not, the chest being naturally an extremely dull one, and the patient one of the few met with where no deep inspiration could be produced. It is curious that for day's I should have remained in positive doubt as to the existence of pneumonia, and even when extensive consolidation of both lungs was definitely ascertained the other symptoms present did not support it. Note particularly the pulse and respirations. It was not until the seventh day after admission that I was beginning to feel anxiety about the lungs, and during the whole of this time the respirations were only i4 per minute. On the fifteenth day the patient was brighter, better, and feeding well. On the eighteenth day there was a relapse, and on the twenty-fifth day he died.

Pulse, Respiration, and Temperature. The pulse for the first and second weeks was natural to the attack, but during the third and part of the fourth week, at a time when extensive changes had occurred in the lungs, it was only between 40 and 50 beats per minute, and of good character; it only reached 60 and 80 shortly before death. The temperature rose and fell in a very erratic manner; it was only one degree above normal on the seventh day, when the nature of the attack was becoming manifest; it reached normal on the fourteenth and fifteenth days, and was only one degree above normal from the fourteenth to the eighteenth day; it was normal on the nineteenth and 
twentieth days, then rose again during the last week of life. This temperature curve is most unusual and very misleading, especially when taken in conjunction with the respirations. These were from I 2 to I 6 per minute during the entire attack, rising to 24 the day before death.

The Chest Sounds were, as previously mentioned, very indefinite. On the eighth day there was extensive consolidation of both lungs. On the thirteenth day the area of consolidation could be mapped out by the tracheal reflex method. On the twentieth day the left lung was still solid below, but the right was admitting air to the bottom, though the murmur was decidedly of an oppressed character, and five days later this right lung was again found solid. This feature will be apparent in more than one of the cases recorded in this series. I can offer no explanation of the circumstance.

Outlines of Post-mortem Examination. A very large abscess containing necrotic tissue in the middle of the right lung, multiple abscesses in the anterior lobe, and considerable consolidation of the inferior third. In the left lung there was no abscess, the anterior lobe was normal, but there was extensive hepatisation of the inferior lobe.

Remarks. This was a very misleading case. (I) The physical examination of the chest, respirations, and temperature gave no assistance in determining the nature of the attack in the early stages, and neither respirations nor temperature gave any help in prognosis throughout the entire case, while the pulse-rate of the third week was positively misleading. (2) The single lung abscess was the largest I have seen. I did not determine its existence during life, though its position was quite favourable for diagnosis. It could have held about half a gallon of liquid. (3) It is interesting to notice that that most fatal of places for multiple abscesses, viz., the anterior lobe, was quite normal in the left lung, and further, that there were no abscesses in any part of this organ.

\section{Case VII.}

Short History. This horse was admitted off feed, the pulse and respirations being normal, the temperature $102^{\circ}$, the chest clear. The temperature steadily rose up to the fifth day, at which time changes in the lung began. By the sixth day pneumonia of the right lung was established. On the eighth day the left lung became affected. The case lived for twenty-seven days.

Pulse and Respirations. During the first week the pulse was from 40 to 48 , the breathing frequently only I 2 or I4. During the second week the pulse was generally 60 , while the respirations were never less than 30 . During the third week the pulse was generally 48 and the respirations 24 , while in the last week of life they were as high as during the second.

Chest Sounds. The chest sounds were as follows. On the day when the lung became suspected there was a modified or oppressed respiratory murmur, below, the air was not entering as freely as it should do, there was an increased murmur in the healthy portion of lung; on deep inspiration there were no crepitations, and the oppressed respiratory murmur caused by the deep inspiration rapidly died away. On the day pneumonia became firmly established, viz., the sixth, hardly any air was entering the affected lung, no crepitations, 
but occasional expiratory squeaks. On the eighth day the left lung became affected like the right, excepting that bronchial breathing was already found. On the ninth day there was a remarkable extension upward of the lung trouble ; the bottom of both organs was absolutely solid, only faint squeakings being heard even at deep inspiration; the bronchial breathing had gone from the left lung. Tenth day, both lungs absolutely blocked for two-thirds, and yet the percussion note was remarkably resonant, excepting at the lower third. No bronchial breathing or tracheal reflex present. On the thirteenth day both coarse and fine squeakings were heard over the middle of the right lung; the squeakings were both inspiratory and expiratory, but generally inspiratory. On the fourteenth day the squeakings were heard all over the lower third of both chests; these squeaks and coarse bubbles were very marked about the centre of each lung, and were present during both inspiration and expiration. Swallowing bronchophony could also be heard. Twenty-third day, the râles described on the fourteenth day were now more marked and coarser, resembling croaking and grunting; no bronchial breathing, and the chest quite resonant on percussion in spite of the complete consolidation of the lower half of each lung. Twenty-fourth day, air entering all over the left chest, a remarkable change from yesterday. No air entering the lower twothird of the right chest, and the grunting, bubbling, and squeaking râles over the middle of the lung were marked. These places are regarded as the site of abscesses. Both chests clear on percussion, but swallowing bronchophony can now be heard. Twenty-fifth day, air still entering the left chest, but at first part of upper third coarse râles were heard both at inspiration and expiration, and there were loud moist crepitations in this region. It is believed that this lung is breaking down. The squeaking râles in the right chest are still present, and in addition loud moist crepitations over nearly the whole of the lung. Twenty-sixth day, the left lung contains only one affected area, and here the râles are converted into coarse bubblings, heard both at inspiration and expiration. In the right chest the entire lung is practically occluded, loud liquid bubblings are heard over the area previously indicated as necrotic, and grunting bronchophony is now present. Twenty-seventh day, found dead in the box, the place covered with blood, owing to hæmorrhage from the lungs, which evidently was the immediate cause of death.

Outline of Post-mortem Appearances. The anterior lobes of both lungs riddled with abscesses; abscesses over the middle third of right lung, where indicated during life, many of them situated in the middle of comparatively healthy lung tissue; another large abscess high up at the posterior part of the lung which was not detected during life. Both lungs at their inferior third were hepatised. The blood-vessel had burst in one of the pus cavities in the anterior lobe of the left lung. Pleura normal.

Remarks. Note (I) The delay in the onset of pneumonia. (2) The low pulse and respiration-rate during the first week. (3) The delay in the attack of the right lung. (4) The remarkably clear percussion note. (5) The commencement of the lung necrosis on the fourteenth day. (6) The extraordinary change in the left chest on the twenty-fourth day, unexplained by post-mortem examination. (7) The rupture of the pulmonary vessel. (8) The unusually long period the patient lived. 


\section{Case VIII.}

Short History. This horse on admission presented no definite condition indicating pneumonia, but in thirty-six hours these appeared, both lungs being affected. The patient fed well. The case lived nearly thirteen days, and for the last three days of life was in great respiratory distress, getting up and down constantly during this time. I never before saw such long continued distress.

Pulse, Respirations, and Temperature. The pulse and respirations during the first week were normal to pneumonia-a pulse of 60 and respirations 30 , but during the second week the pulse-respiration ratio was very curious, viz., a pulse of 72 , and respirations of 60 , or a pulse of 86 , and respirations of 74 . The temperature on admission was $107^{\circ}$, with a fall to normal on the morning of the second day, but with a rise in the evening to $104^{\circ}$. I have observed this drop to normal and subsequent rise on more than one occasion.

Chest Sounds. First day, oppressed respiration. Second day, absolute dulness of both lungs, lower third. Third day, extension of disease upwards; a deep inspiration producing wheezing in the affected area. Sixth day, squeaking râles are heard at intervals all over the chest. Seventh day, bronchial breathing behind left elbow. Tenth day, over the middle of the right lung is an area of intense squeaking râles, heard both at inspiration and expiration; very little bubbling, though some is present. I regard this as an abscess forming. In the left lung is a large area of bronchial breathing behind the elbow. Eleventh day, increase in the squeaking and grating area in the middle of the right lung, and tubular breathing below. Considerable squeaking and cooings in the left lung at different parts. Twelfth day, bubbling and squeaking râles as before, hepatisation of both lungs below, tubular breathing in both chests behind the elbow.

Outline of Post-mortem Appearances. Both lungs contained innumerable abscesses in the anterior lobes, the lower third of both lungs firmly consolidated, and where the squeakings and bubblings were heard during life were large abscesses, many of which were some distance from the inflamed area. An attempt to inflate the lungs after death failed on account of a leak into the pleura.

Remarks. Note (I) the strange pulse-respiration ratio during second week. (2) The sudden drop in the temperature on second day. (3) Commencing necrosis of lung on the tenth day.

\section{PLEURO-PNEUMONIA.}

\section{Case I.}

Short History. Admitted with sore throat and high temperature; on the fourth day pneumonia occurred, the pulse went up to 80 , the respirations to 36 . With very little variation the symptoms lasted two weeks, the case dying on the nineteenth day after admission. Effusion occurred as early as the third day following the acute symptoms.

Outline of Post-mortem Examination. Revealed abscesses in both lungs, abundant putrid effusion, lungs adherent superiorly to chest wall and freely plastered with lymph. 


\section{Case II.}

Short History. This was an extremely rapid case, admitted one evening with a temperature of $104^{\circ}$, pulse 45 , respirations 20 . I did not see the patient on admission, so do not know the state of the chest. The following morning there was marked friction over left lung, great collapse, hardly any pulse, which was 60 to the minute, short abdominal respirations 36 . On the third day the case was dying, the pulse 80 , respirations 48 ; in the afternoon the patient got down and was unable to rise even with assistance. He showed signs of what appeared to be abdominal pain, was pulseless, and even the heart sounds could not be distinguished. Convulsions occurred at intervals, and he died the same night, fifty-two hours after the attack.

Outline of Post-mortem Appearances. Effusion of blood-coloured serum into the left pleural sac only, membranes on the lung but none on chest wall. Left lung inferior third hepatised. Right lung congested but otherwise normal. Pericardial sac over an inch in thickness from serous exudation the result of inflammation, and there was some fluid within the sac.

Remarks. (I) A curiously sudden case. Why the collapse? The right lung was perfectly fit to carry on the needful work for a long time, and this sudden case is very suggestive of a poison. (2) There was no abscess in these lungs, nor indeed should we expect such within so brief an illness. (3) The effusion was not diagnosed during life, and it is one of the very few cases met with where the exudation was single sided.

\section{Case III.}

Short History. This horse was found ill with a temperature of $106^{\circ}$, a pulse of 100 which could not be taken at the jaw, and even the heart-beats indistinct; the breathing 54 to the minute, but strange to say, in spite of this rate, there was not the slightest movement of the nostrils. The chest was quite clear. On the second day there was well-marked pleuritic friction of the right chest, great distress and grunting, and heart sound still very muffled. On the third day the patient fed well, friction still present over right lung, and off fore leg advanced. On the fourth day there was friction over both left and right lungs, and pericardial friction likewise. On the sixth day feeding well, friction sound over both chests gone, and now it is possible to ascertain that both lungs are consolidated below. Ravenous appetite. Seventh day, not so well. Eighth day, no improvement, off fore leg still advanced. Ninth day, worse, great respiratory distress, for which the pleural sac was tapped, but only a little fluid came away. Knowing the pericardium to be affected, and thinking the respiratory distress might be cardiac, the pericardial sac was tapped, but obtained only a little fluid, and on pushing the instrument in deeper obtained an alarming gush of blood. Tenth day, great respiratory distress with dilated nostrils, anxious expression, air entering to the bottom of right chest, which made one think there could be no effusion present, but in this, as the post-mortem will show, I was mistaken, Refuses all food. Tenth day, died.

Pulse, Respirations, and Temperature. The pulse started with Ioo, 
fell on the fourth day to 48 , then rose again, and was at Ioo the last two days of life. Though very oppressed at first, it subsequently became of much better volume. The respirations throughout were between 50 and 60 . The temperature was very high, $106^{\circ}$ being reached three times.

Chest Sounds. While the friction lasted it was very intense; it was of a cracking, creaking character as heard through the phonendoscope, and masked all the lung sounds by its intensity. It is seldom that the sound is so intense.

Outline of Postmortem Appearances. Abundant effusion into both chests, great collapse of both lungs from hydrostatic pressure. The lower half of the chest was plastered with lymph, and the inferior half of the lungs was in a similar condition. The inferior fifth of the right lung and rather less of the left were pneumonic and consolidated, and on cutting into this portion it was found full of abscesses. The remaining portion of lungs was healthy. The diaphragm was coated with lymph from top to bottom. The pericardium enormously thickened and blood stained, while the sac itself held about a pint of blood-coloured fluid. There were two marks in the left ventricle near the apex, indicating where the trocar bad touched it, but neither had penetrated the wall of the ventricle, at which I was surprised, considering the stream of blood which came through the canula.

The horse was a whistler, but the larynx showed no disease.

Remarks. (I) This horse died from slow asphyxia, due to the accumulation of fluid in the chest. I probably could not find the latter owing to the membranes present. (2) Air appeared to be entering to the bottom of the chest on the ninth day, at a time when the lungs were not within several inches of the sternum. The sound is therefore transmitted through the fluid in certain cases, and is most misleading. (3) The pericardial tapping is not a very satisfactory record, but fortunately did no harm. (4) The high pulse and respiration rate of this case is noteworthy. (5) Pointing one or both fore legs is not uncommon early in pleurisy.

\section{Case IV.}

Short History. Admitted with a temperature of $106^{\circ}$, pulse 70 , respirations 44 . The patient walked to the infirmary, only a few yards distant, and was almost prostrate, so intense was the sudden depression. The animal grunted, but nothing definite could be made out of the lungs until the evening, when it was evident both were affected. Heart sounds were feeble. On the second day there was great collapse, almost pulseless; temperature in the evening $107^{\circ}$, pulse IOO, respirations 48 . Third day, pulse 100 , respirations 40 , temperature $105^{\circ}$. In the left lung little or no air is entering below, and where it is cooing is present. The right lung is fairly clear. Died sixty-two hours after admission.

Outline of Post-mortem Examination. The whole pleural membrane of both sides minutely injected and ecchymosed, but no membranes. The pericardium was a sight, simply one big splash of blood extravasation, beneath which was a thick gelatinous effusion. The left lung was pneumonic, especially superiorly (which is rare), and possessed the same marbling as a pleuro-pneumonic lung; in the left 
sac was a little fluid. The right chest contained volumes of bloodstained fluid, the right lung pneumonic below and hepatised, and there was also marbling of the superior lobe of the lung, as in opposite chest. The heart was quite extraordinary; it was speckled with blood spots, the fat gelatinous, and the pericardial sac contained much fluid. The trachea and bronchial tubes were nearly black, and even the tissue outside the trachea odematous. In the pharyngeal glands was some creamy pus, while the guttural pouches were purple.

Remarks. (I) A very virulent case affected from the fauces to the diaphragm. (2) The effusion was undiagnosed, and it will be noted that air was heard entering to the bottom of the right chest on the last day of life, though post-mortem examination showed that no air could have entered. (3) The heart lesions were curious, during life the sounds were extremely feeble.

\section{Case $V$.}

Short History. Admitted with pleurisy and pneumonia, the physical signs of the disease being very inarked; there was an entire loss of appetite, and very rapid thumping action of the heart, and in this way the case continued up to the tenth day, when some improvement in the symptoms was manifest; on the sixteenth day there was a distinct relapse, the breathing indicated effusion, and the chest was tapped; already the fluid had a faintly foetid odour; on the nineteenth day he died.

Pulse, Respirations, and Temperature. Nothing to record, quite normal to the case. The pulse was at times peculiar in character, some beats being full and good, and others being so indifferent that the artery appeared almost empty. The pulse was occasionally intermittent. The heart's action against the chest wall was most violent from the first.

Chest Sounds. On admission well-marked pleuritic friction of the left chest and dulness of left lung below; no friction in right chest, but lung dulness well marked below. On the second day the consolidation of the lungs had increased, still friction over the right lung, but none over the left. On the fourth day the friction had disappeared, a little air was entering the left lung below, but none in the right. On the fifth day no air could be heard entering the left lung below, the right still solid, fine tubular breathing could be heard in the left lung for the first time. On the thirteenth day bubbling was heard over the larger bronchi on the left side, and tubular breathing below in the right lung; no respiratory sound whatever below. On the fifteenth day tubular breathing appeared in the right lung at the bottom, left lung no change. On the seventeenth day, though abundant effusion existed in the chest, tubular breathing could be heard at the bottom of the chest in the entire absence of lung, showing that this sound was transmitted through the fluid. The abdominal breathing was characteristic of effusion.

Outline of Post-mortem Appearances. Pleurisy of the lower half of the chest wall and the whole of the pulmonary surface; not a single false membrane, the pleura being simply scarlet and maroon. Anterior lobe of right lung turned back, and glued to the outer surface of the upper third of the lung. This right lung was carnified from hydrostatic pressure, and contained a large abscess close to the bronchi, but I could see no 
pneumonia; the right lung was also attached to the ribs. The left lung was attached to the chest wall and to the diaphragm; it contained in its interior an abscess like the right lung, was carnified from pressure, and had scattered through it islands of pneumonic patches, more in the form of hæmorrhages, but no attempt at hepatisation. The pericardial sac was black and scarlet, and greatly thickened, but no false membranes.

Remarks. (I) There are some peculiar features in this case; one important one is the absence of membranes in acute pleurisy; none were found anywhere, not even in the fluid from the chest. (2) Note the violent action of the heart and the pulse peculiarities, such are not uncommon as the result of the pericarditis. (3) Observe that there was not a single patch of hepatisation in these lungs, and that one at least would have been normal had it not contained an abscess and become carnified from pressure. (4) Note that respiratory sounds were heard where no lung existed, and also that the solid lung I expected to find did not exist.

\section{Case VI.}

Short History. On admission this horse was found to have pleurisy on the left side, the pulse 92, could hardly be felt, and the heart ticking like a clock. The lungs appeared clear, at least a normal murmur could be distinctly heard through the friction. Second day, pulse better, right chest still clear. Third day, friction gone from left chest, but the lung is now affected, the respiration being very oppressed; right chest not quite satisfactory. Fourth day, marked respiratory distress, feeding badly, extension of lung trouble. Fifth day, there has been a peculiar pulse-respiration ratio, which is extremely marked now, viz., a pulse of 50 , and respirations of 40 . The chest is admitting more air through the affected portion. Sixth day, better, feeding, and brighter. Eleventh day, has been doing well up to to-day, when the appetite has failed; the right chest is extremely wooden on percussion, and no air entering its lower half; air enters the left lung, but very imperfectly. Thirteenth day, evidently doing badly, great loss of condition, improvement in pulse, peculiar ratio still present, chest sounds no change. Sixteenth day, the respirations are characteristic of effusion, bronchial breathing present in right chest behind the elbow. In the left chest air can be heard to the bottom, yet the chest contains a considerable amount of fluid as proved by tapping. Seventeenth day, died.

Pulse, Respirations, and Temperature. The pulse was generally 6o, excepting the first and last days of life, when it was 90. 'The respirations were between 30 and 40 , so that the pulse-respiration ratio was very singular; for example, a pulse of 50 and respirations of 40 . The temperature was seldom high, and on the tenth day reached normal.

Outline of Fost-mortem Examination. Only the lower half of the chest affected with pleurisy, the upper part quite free; diaphragm affected. No membranes of any lind found in the chest, but the affected pleuræ were engorged, even in some places to blackness. The right lung was pneumonic below, but the left was free; both lungs were greatly compressed from hydrostatic pressure, and carnified in consequence. In the left lung, close to the bronchus, was a large abscess, but the surrounding lung tissue did not look pneumonic. 
There was perforation of the pleura, so that the lungs could not be distended. The pericardial sac was thickened and quite black externally, and even the internal surface was slightly affected, which is very rare. Both lungs and diaphragm were adherent to the ribs in many places.

Remarks. (I) This case presents a feature very common in hydrothorax, viz., a decided improvement in the symptoms followed by a relapse. (2) The pulse-respiration ratio is very curious; it cannot be accounted for. (3) Observe, again, that air appeared to be entering to the bottom of the chest on the last day of life, though the thorax was full of fluid.

\section{Case VII.}

Short History. This horse was admitted with a temperature of $104^{\circ}$, no disturbance of the breathing and but little of the pulse. The chest was perfectly clear until the evening of the fourth day, when a little squeaking was heard behind the left elbow, though the air was entering the lung, and the pulse, respirations, and temperature were nearly normal. On the fifth day the temperature rose $4^{\circ}$, and on the evening of this day undoubted lung trouble was present, both sides being affected, and strange to say the upper part of the lung was more involved than the lower-one of the very rare exceptions met with. The pulse was good, the respirations of a peculiar snorting type not uncommon in acute chest trouble, and the near fore leg was advanced, resting on the toe. Eighth day, no change, pulse particularly good. Tenth day, worse, grunting, and painful cough, extension of disease in both lungs, no appetite. Twelfth day, worse, great depression, grunting, no appetite, but pulse wonderfully good. Fourteenth day, a pleuritic patch discovered, patient much worse, pulse failing. Sixteenth day, no change, snorting respiration still present; it is due to the false nostril being drawn in at inspiration. Eighteenth day, still alive, pulse a mere thread, slight blood-stained discharge from the nostril. Nineteenth day, died.

Pulse, Respirations, and Temperature. During the period of incubation the pulse and respirations were normal ; later the pulse rose to 6o, respirations to 24 , and so remained until a few days before death. The temperature on admission was $104^{\circ}$, and then fell to nearly normal, until the fifth day when it rose to $105^{\circ}$. On the seventh and eighth days it again fell to nearly normal, and never subsequently rose above $103^{\circ}$.

Chest Sounds. On the fifth day both lungs dull in the upper third, squeaking behind right elbow, and no air entering behind the left. Sixth day, chest clearer than on fifth, but the patch of consolidation in the right lung is still present, and on deep inspiration there is squeaking and crackling. Seventh day, left chest clear. Right chest, undoubted pneumonia both behind the elbow and above; pneumonic crackling on deep inspiration and much wheezing. Ninth day, the left lung is now affected, and like its fellow is attacked both above and below. Tenth day, both lungs blocked below for more than onethird, squeakings can be heard on deep inspiration, more marked in the right than the left lung. Bronchophony in left and not in right lung. Thirteenth day, pleuritic friction behind right elbow, no air entering either the top or bottom of the lungs, the middle portion only 
carrying on the work. Fourteenth day, tubular breathing in left chest below, and grunting bronchophony ; tracheal reflex well heard. Extension of friction behind the right elbow. Eighteenth day, left lung completely blocked above and below, yet on percussion the note above is clear, though no air is entering the lung. In the posterior portion of this upper region is a part where at each inspiration a peculiar sound is heard, such as might be produced by dropping a stone into a well. On the chest chart I marked this as probably an abscess, though I had never heard the sound before. Post-mortem examination showed that there was no abscess. In the right lung the lower third was solid; above this an area of fine squeaking bubbling crepitations. In the upper-third were also crepitations.

Outline of Post-mortem Appearances. The lungs could not be inflated, showing perforation had occurred. Right lung anteriorly was sold from top to bottom, the only breathing area was the upper and posterior part; no abscesses. Left lung one solid mass, though not so firm as the hepatisation of the right lung; in the anterior lobes were innumerable abscesses. Marked pleuritic roughness over right lung and chest wall, but no fluid in the chest.

Remarks. (I) This is a very exceptional case; it is seldom that an entire lung is destroyed by pneumonia, and certainly the left was in this patient. (2) It is curious that the abscess production should have been so limited.

\section{Case VIII.}

Short History. This is an extremely interesting case from the rapidity with which the disease ran its course, killing the patient in three days. This horse on going out to exercise one morning was observed to be unusually fit and well. The exercise consisted of walking for two hours at quite a moderate pace; the animal returned from it in such a state of collapse that it was with difficulty it could be dragged to a loose-box, and this will be readily appreciated when it is stated that the temperature was $108^{\circ}$, the pulse 108 , and the respirations 40. There was the most intense depression, the left chest presented one small patch of pleurisy, and the respirations below were of an oppressed character. In the right lung the inferior third was partly blocked, and pneumonia present. This condition of the chest was in itself insufficient to account for the very acute character of the symptoms. On the second day he was obviously dying, and there was a large pleuritic patch at the upper and posterior third of both lungs; great consolidation of right lung for more than one third, and well-marked changes occurring in the corresponding part of its fellow; in addition the patient was shivering and blood coming from the nostrils. On the third day the pulse was I28, counted at the heart, nothing could be taken at the jaw. The chest symptoms remained unchanged, excepting that there were marked fine crepitations over the middle of the left lung. There was great distress, pawing with the fore legs, resting the head anywhere, against the wall or the manger, moving round the box and unable to see where he was going, and other symptoms pointing to delirium. Occasionally there would be severe attacks of dyspnoa, the tongue would be protruded and become livid, and breathing would take place through the mouth; these symptoms passed off as rapidly as they came on. The breath- 
ing did not indicate effusion, though I might have suspected it from the respiratory distress, but I hardly expected any serious effusion in three days. He died on the third day.

Pulse, Respirations, and Temperature. The pulse on admission was 108, it never fell lower than 80 , and was more often IoO. The breathing never fell below 36 , and was 48 before death. The temperature of $108^{\circ}$ on admission in the morning fell to $106^{\circ}$ at 5 P.M., and $104^{\circ}$ at 8 P.M. On the second day it again rose to $106^{\circ}$.

The Chest Sounds were not very satisfactorily made out; the lung which appeared the least affected turned out on post-mortem examination to be the worst, and again I met with that feature which I cannot too frequently record, viz., at one examination and for a brief time one lung appearing comparatively clear, though at the previous examination a few hours before it was blocked below, and the same condition found a few hours later. Were it not that I have met with this curious feature many times I would put it down to an error of observation. It only shows that we cannot examine the chest too frequently. During the last few hours of life air could be heard entering to the bottom of the chest though the cavity was full of fluid. I am noting this condition wherever in this series it has been observed, as the symptom has its practical bearing-the respiratory murmur is transmitted by the fluid.

Outline of Post-mortem Examination. Both chests full of bloodcoloured serum, containing clots of fibrin-like curds. Acute pleurisy of lung, chest wall, and diaphragm. Left lung just behind the heart was swollen and firm, and the mass of a greenish colour, indicating gangrene. On cutting into it, it proved to be little else than one vast blood clot due to hæmorrhage; above the apoplectic area the lung was greyish and pneumonic. The whole of this diseased condition was almost entirely limited to the inferior third of the organ. The right lung was affected with red hepatisation but no apoplexy. No abscesses could be discovered in the lungs, and the anterior lobes were normal, which is very rare. The lungs distended under inflation. The pericardial sac was enormously thickened and discoloured from inflammation.

Remarks. (I) Note the rapidity of the invasion and the rapid progress of the case. (2) The intensity of all the symptoms excepting those of the chest, which though severe were not sufficiently so to account for the collapsed state of the patient. (3) It is possible that cases of this sort are different from ordinary pneumonia. (4) It would certainly appear to be a virulent poison which can affect such a relatively small area, and cause such urgent symptoms and death.

\section{Case IX.}

Short History. Admitted in the evening "blowing" and occasionally lying down. The pulse peculiar, swells under the finger, then dies away; marked laryngeal respiration; both lungs were affected and the pleura on the right side. On the third day the pulse was excellent, feeding well, temperature and pulse dropped, and every indication of improvement. Everything went on well, temperature, pulse, and respirations normal until the ninth day, when the temperature rose, and all the old symptoms returned; from this to the 
thirteenth day there was a gradual increase in all the symptoms, and death then took place.

Chest Symptoms. On admission both chests below were very oppressed, and on deep inspiration there were crepitations. On the second day the chest was clearer and no crepitations, but on the evening of that day well-marked pleuritic friction existed; this had disappeared the following day, and only an extremely oppressed respiratory murmur remained. On the ninth day there was a relapse ; the area of lung dulness became much greater, there being no respiratory murmur below even on deep inspiration. Tenth day, tubular breathing present behind left shoulder, other symptoms as before. Eleventh day, the dulness on percussion is intense, but the respirations do not indicate effusion. Swallowing bronchophony present in left chest. Twelfth day tubular breathing all over the bottom of the left chest (though post-mortem examination showed it to be full of fluid), respiratory dulness everywhere, pleuritic friction above. In the right chest pleuritic friction above, entire absence of any murmur below, no tubular breathing (yet this chest was full of fluid as on opposite side). Thirteenth day, tubular breathing all over the bottom half of both chests.

Outline of Post-mortem Examination. Both pleural sacs from top to bottom and front to rear were plastered all over with fibrinous lymph. There was an abundant putrid effusion, entirely unrecognised during life though repeatedly looked for. The right lung was pneumonic at the inferior third behind the heart; it contained two large abscesses. The left lung though covered with lymph was perfectly free from pneumonia. The anterior lobes were quite healthy. The collapse of the lungs from hydrostatic pressure was remarkable. The percardial sac was enormously thickened from inflammation.

Remarks. (I) Note the relapse after what appeared to be complete convalescence. (2) Tubular breathing in spite of effusion. (3) The freedom of the right lung from pneumonia in spite of the acute pleurisy.

\section{Case $X$.}

Short History. Admitted with well-marked pleuritic friction of the left chest, with an exceedingly bad pulse of 60 , high temperature, but respirations only 14 . On the second day the friction was still present, and through it the respiratory murmur could be heard; behind the heart the friction was almost continuous, due to pericarditis; in the right chest behind the elbow was a dull patch. Third day most intense friction in left chest, at times almost crackling; there is a small friction patch behind the right elbow. There is no appetite, the temperature higher, pulse 72 , and respirations 24 . Fourth day, in the evening the friction had disappeared. On the fifth and sixth days there was a decided improvement, pulsc and respirations fell considerably, but no important fall of temperature. On the seventh day there was a relapse, the pulse and respirations rose ; the left chest was very dull on percussion, and tubular breathing was present, while in the right chest there was increasing dulness behind the elbow. On the eighth day the lower half of the left chest gave a very oppressed murmur, and in addition there were very coarse harsh râles present and also grunting. On the ninth day there was a blood-tinged discharge from 
the nostrils and foetid breath. The appetite had been satisfactory since the fifth day, but had now failed. Eleventh day, foetid grumous discharge from nostrils. The chest examination revealed the inferior part of the left chest clear on percussion; and occupying the middle and lower third of the lung was an area full of moist râles something like the bubbling of a boiling pot. It is considered that this is due to an abscess; in the right lung there is a place behind the elbow where bubbling is occurring, and this is also regarded as an abscess. Twelfth day, died.

Outline of Post-mortem Examination. Both pleural sacs and diaphragm intensely inflamed. A very large abscess existed in the left lung where located during life, and it freely communicated by numerous holes with the pleural sac; the lungs consequently could not be inflated. The right lung was one-third consolidated, but contained no abscess. The bubbling heard on this side must therefore have been reflected from the opposite lung. The pericardium was severely affected.

Remarks. A single large abscess (probably resulting from the fusion of several small ones) in the lung is very rare

\section{PLEURISY.}

\section{Case 1 .}

Short History. Admitted very dull, temperature $105^{\circ}$, pulse 80, very oppressed and intermittent, respirations 25 . No friction on auscultation, but there is almost entire absence of air from the base of the lungs. On the fourteenth day effusion occurred, but, unlike what generally takes place in these cases, there was no indication of improvement prior to effusion.

On the fourth day the temperature fell to $\mathrm{IO} 2^{\circ}$, but the pulse and respirations were still high, though the volume of the latter had improved. The temperature taken as a whole was high throughout the attack, it being nearly always $104^{\circ}$. The pulse was peculiar; after the first day or two it became large and full, and remained so throughout the attack. The breathing was uneven, short or catchy, and the cough was strong, the latter symptom being misleading and unusual.

Outline of Post-mortem Examination. General pleurisy of both sides-lungs and ribs. The lungs were collapsed from hydrostatic pressure, and were free from disease if we except an abscess between the bronchi, which I think was only a gland and actually outside the lung. The collapse of the lungs was remarkable ; there was practically no lung below a horizontal line drawn on a level with the base of the heart. The pericardium was acutely inflamed and thickened. There was aneurism of the mesenteric artery (parasitic), which probably accounted for the pulse during life. There was an abundant exudation in the chest; it was turbid but free from odour.

\section{Case II.}

Short History. This horse had been standing in a railway siding for some hours on a very cold day, and in the evening he was attacked with a shivering fit. The chest was dull, but there was no friction, the temperature $106^{\circ}$, pulse 60 , respirations 36 . The breathing was short 
and painful, and later on grunting. The case did well for a few days, temperature falling to $102^{\circ}$, the pulse to 40 , and the appetite being enormous. On the seventh day there was a relapse, but there was no indication of effusion. The pulse was good, and the appetite remained excellent. On the thirteenth day the respirations indicated effusion, the chest was tapped and a putrid fluid obtained; the pulse was I00, the respirations 36 . He died on the sixteenth day, feeding to the end.

Outline of Post-mortem Appearances. The entire pleural sacs were covered with dense membranes, and superiorly the lungs were adherent throughout their entire length to the ribs, and had to be dissected away. The lungs were also adherent to the diaphragm. The lungs were perfectly healthy, no trace of pneumonia; the inferior lobes were collapsed and carnified from hydrostatic pressure, but the organs were quite healthy. Careful search was made for an abscess to account for the putrid condition of the chest fluid, but none was found. The pericardium was intensely affected; there were many gallons of putrid effusion in the chest.

Remarks. (I) Observe the relapse on the seventh day. There is no doubt that at this time effusion commenced, though it was not recognised until later. (2) The entire freedom of the lungs from disease shows that cases of pure pleurisy are possible, though very rare. (3) I never before or since saw a putrid effusion in the chest which was not due to infection by an abscess.

\section{Case III.}

Short History. Admitted with intense pleuritic friction over the left chest, and a smaller patch behind the right elbow. It was the most intense friction I have ever heard, and over the region of the heart was synchronous with the heart-beats, showing that pericarditis was also present. The temperature was high, and the horse very ill. Second day, friction still present. Third day, friction gone, chest excellent; a modified though distinct murmur can be heard over the base of the lungs, appetite poor. Fourth day, friction returned in left chest, while the right chest was very dull behind the elbow. Up to the ninth day the case appeared to make fair progress, but the temperature was very variable, and though the pulse did not fall below 50 the respirations dropped to 12 . On the tenth day the dulness of the chest was found to extend half-way up on either side, and though the breathing did not indicate it effusion was suspected, as the line of dulness was very sharp, and oedematous swellings of the sheath and brisket were present-a fairly constant symptom in hydrothorax. Fifteenth day, has been doing fairly well up till to-day, but now very dull. The chest sounds on percussion are of a wooden character, and no air enters the lower half of both lungs; the breathing does not indicate effusion. Seventeenth day, relapse, greater distress in breathing, which has risen from 14 to 25 , so tapped the right chest and drew off 5 quarts of serum. Eighteenth day, tapped left chest as the breathing was still distressed ; drew off 6 quarts, and relief was obtained. Twentyfirst day, tapped both yesterday and to-day, Io quarts removed; tubular breathing is present over the lower third of each chest, while in the right chest superiorly there is a very oppressed respiratory murmur. Twenty-fourth day, feeding well, the tapping of to-day had a faint odour, the breathing does not indicate hydrothorax. Twenty- 
seventh day, slight improvement in general symptoms, tapped left chest to-day, and the respirations were somewhat deeper, the fluid from this side was sweet. Thirty-fourth day, by this date both chests contained fotid fluid, but the patient has managed to hang on in the most remarkable manner; there is also a nasal discharge with a fotid smell. Died.

Temperature, Pulse, and Respirations. The temperature started at I06 and for the first week there were considerable daily variations, as much as three degrees between the morning and evening temperature. During the second week the temperature fell, reaching normal on the fourteenth day. It rose on the seventeenth day owing to the relapse, but during the fourth and fifth weeks of the disease it remained very little above normal. The only point in connection with the respirations is that for the first two weeks they varied from I 2 to I4, which is extremely low for acute pleurisy. On the fourteenth day, with a temperature of $100^{\circ}$, pulse 48 , and respirations 12 , one had every reason for believing that recovery would possibly follow.

Outline of Post-mortem Examination. Both lungs collapsed to an extreme degree; neither of them extended below the base of the heart. The inferior half of the chest was filled with putrid fluid. The right lung was firmly fixed to the ribs above by adhesions, carnified from compression, and contained anteriorly one abscess. The left lung had no membranes upon it, and with the exception of a little pneumonia along the lower edge and a small abscess it presented nothing of importance; it was of course considerably reduced in size by the compression to which it had been exposed. The pericardium was enormously thickened. At least one of the bronchial lymphatic glands was in a state of suppuration. The lungs could not be inflated owing to perforation.

Remarks. This case is chiefly remarkable for (I) the length of time it lasted, especially after the fluid had become putrid. (2) The absence of any characteristic hydro-thorax breathing. (3) The entire freedom of one lung from pleurisy though the chest-wall was affected. (4) It is nearly but not quite a case of pure pleurisy; there was absolutely no pneumonia in spite of the two abscesses, with the exception of a short narrow strip along the lower border of the left lung. Properly speaking the case should be classed with the pleuropneumonia series, but it so narrowly escaped being one of pure pleurisy that I have included it here.

\section{Case IV.}

Short History. This horse came in with severe rigors, high temperature, and suspiciously dull chest ; the pulse was good and full, and therefore misleading. On the second day pneumonia was undoubted. On the third day the pulse was very small, temperature high, grunting, and patient extremely ill. On the fourth day worse, no appetite, breathing very laboured. Suspect effusion though friction has never been detected, and pleurisy not hitherto suspected. Fifth day, tapped the right chest and got a little blood-stained fluid; tapped the left chest in two places and obtained nothing; towards the evening there was very great distress, sweating, and lying down. Sixth day, intense respiratory distress; tapped both sides and drew off many quarts of blood-stained serum having a distinctly foetid 
odour. During the whole day there was great distress, and death occurred in the evening. The heart sounds before death were single.

Pulse, Respirations, and Temperature. On admission the pulse was 56 , and it did not rise to So until the end of the fourth day; the breathing throughout was rapid. The temperature was $106^{\circ}$ on admission, and only once below $105^{\circ}$.

Chest Sounds. First day, dulness of both chests below. Second day, dulness extending up, and even at deep inspiration nothing can be heard. Third day, no air whatever entering lower half of both chests, tubular breathing in left chest. Fourth day, tubular breathing over the entire lower half of both chests, though at this time the cavity contained a considerable amount of fluid.

Outline of Post-montem Examination. The lungs could not be inflated, but there was a rupture of the pleura in the anterior lobe of the right lung. The pleural sacs did not communicate; the fluid in the right was turbid, that in the left quite clear. There was general pleurisy of both cavities, but the lungs though compressed and carnified as a result were otherwise healthy.

Remarks. (I) It is curious that the advent of pleurisy should be ushered in by symptoms which pointed to the lung and not the pleura. Pleurisy was not suspected until effusion occurred. (2) It is strange no fluid was obtained when the chest was tapped on the fifth day, though an abundance was obtained on the following day. (3) The tubular breathing heard at the bottom of the chest was the sound conveyed through the fluid, there being no lung here whatever. (4) The case is an example of pure pleurisy.

\section{Case $V$.}

Short History. This case was admitted with pleuritic friction of the left chest, and a suspicious dulness of the lower lobe of the right lung. The pulse was 60 , and the respirations only i2. On the second day the temperature fell to $\mathrm{IOI}^{\circ}$, the respirations still remaining at $\mathrm{I} 2$. On the third day the area of pleuritic friction in the left chest was greater, and there was also an absence of respiratory murmur from the inferior lobe; in the right lung well-marked pneumonia was present, accompanied by tubular breathing; there was also a considerable rise in temperature, the appetite was poor, and an amber-coloured discharge, very common in pneumonia, trickled from the nostril. On the fourth day the temperature was still rising, now reaching $106^{\circ}$, the respirations had gone up to 28 , the friction in the left chest had disappeared, while the right chest remained as before. On the seventh day there was an increase in the pneumonia of the left lung and tubular breathing, the right lung had undergone no change, the horse was very ill, no appetite, and as the line of percussion dulness of the right chest was very marked effusion was suspected. Eighth day, respirations were distressed, tubular breathing now heard all over the left chest, and to a,greater degree in the right chest; the breathing being more laboured the suspicion of effusion was increased. Ninth day, tapped the left chest, and removed I 5 quarts of putrid serum. Thirteenth day, several tappings have been performed on both chests since the last entry, all of these being putrid; great distress in the breathing, and the latter is not characteristic of effusion. Fifteenth day, died early in the morning. 
Outline of Post-miortem Examination. There was practically no pneumonia, at any rate no hepatisation; the right lung was compressed to an extraordinary degree, and had suffered from carnification accordingly. In it there was one small abscess about the middle; in the left there were several small abscesses immediately behind the heart, and this lung was only compressed to a trifling extent. In each lung it was the carnified portion which contained the abscesses. There was yeneral pleurisy of both lungs and chest wall, though in the right chest the wall was not affected the whole way up. Both lungs were adherent in several places, and the anterior lobes were firmly adherent to the pericardium. The pericardium was severely affected.

Reinarks. (I) Note the complete collapse of the right lung (it was only $\mathrm{I} \frac{1}{2}$ inches in thickness at its thickest part), and the very partial collapse of the left. There must have been immense pressure exercised on the mediastina, and further, it is clear that the two pleural cavities did not communicate. (2) The absence of pneumonia and the presence of abscesses in the lung is peculiar; I cannot explain the fact, but it may yet be shown that carnified lung is as suitable a site for necrosis as hepatised lung. On the other hand, as in a previous case of this series, the fact of any abscess being in the lung may preclude it from falling under the head of pure pleurisy. However, I leave it and other cases provisionally under this head.

\section{Case VI.}

Short History. This horse was seized with a sharp rigor, and on examining the chest nothing could be made out for three or four hours, when a patch of pleurisy was very evident behind the right elbow; by the evening there was intense friction all over the bottom of the left chest, the pulse was very oppressed, and though the temperature on admission was only $1016^{\circ}$, it rapidly rose to $105^{\circ}$; pulse 80 , respirations 40 . Second day, all friction gone; both chests, but especially the left, extremely dull below; temperature fallen and patient better. On the fourth day the temperature fell to normal, pulse 50 , respirations $\mathrm{I} 2$, and this satisfactory condition lasted until the seventh day, when a relapse took place, temperature, pulse, and respirations rising, but strange to say the chest symptoms were apparently trifling; both sides were resonant on percussion, and there was only slight oppression of the normal murmur. As effusion was suspected, the greatest care was exercised in the examination of the chest. On the ninth day the patient was feeding badly and very ill, the pulse was 80 , the respirations 28 , but though the effusion is gravely suspected, the percussion note is still clear, in spite of the fact that both lungs give no indication of air passing through them below, and tubular breathing is present behind the right shoulder. Tenth day, the left chest is quite dull on percussion, the right chest resonant, tubular breathing is present in both, intense friction can be heard in the upper third of the left lung, while in both no air can be heard passing through the lower half. The left chest was tapped, and the serum obtained had a slight odour. Eleventh day, tapped the right chest and obtained putrid serum. Twelfth day, died.

Outline of Post-mortem Appearances. The lungs could not be inflated, therefore pneumo-thorax existed. In both lungs a few small abscesses were found, but otherwise they were healthy; no pneumonic 
tissue surrounded these abscesses, and they were, I think, of pyæmic origin, for between the lungs and diaphragm, on the right side, were three abscesses. Both lungs were much collapsed from pressure and carnified. There was general pleurisy of costal and pulmonary membrane, the entire chest being affected, and also the pericardial sac. Both chests contained a quantity of turbid fluid.

Remarks. (I) This is a somewhat singular case, owing to the chest sounds. I can offer no explanation of the observed phenomena. (2) I have recorded the case as one of nearly pure pleurisy; again exception may be taken to this on account of the abscesses in the lungs, but I think these were secondary, and due to infection from the diaphragm abscesses, for there were none of the ordinary appearances of pneumonia present. On this point the remarks made in previous cases apply. (3) It is curious to observe how commonly relapse follows about the end of a week, especially in those cases which appear to be doing quite satisfactorily ; these symptoms are most misleading, and should not throw us off our guard or induce a hasty prognosis.

\section{SOME INQUIRIES CONCERNING THE LATHYRUS SATIVUS: ${ }^{1}$}

By John B. Wolstenholme, F.R.C.V.S., F.R.M.S.

IT is noticeable in veterinary and medical literature that this legume is at one time referred to as a "pea," and at another as a "vetch," and then again as "these peas, or vetches, to give them their proper title." This seeming ambiguity has induced me to have some fifty plants reared in a good garden in the country, and to refer to several works and papers on the subject. It is the results of these inquiries I wish to bring forward.

In this country in the corn trade the seeds of the lathyrus sativus are known as "mutters." They vary much in colour, being grey, yellow, light brown, dark brown, or nearly black. They are irregular in shape, being compressed and angular, and vary in size; on the average six placed in apposition measure I inch. Lathyrus sativus, the Linnæan name, is the scientific one adopted in France, Holland, and this country. Louden gives chickling vetch as the common British name. Gesse is the French name for the genus lathyrus, and gesse cultivée the name of this particular species (Lamarck). Parkes gives kassaree-dholl as the name by which it is known in India.

Bentham, in his "British Flora," p. I3 I, uses the term pea as synonymous with lathyrus in his heading of the genus. $\mathrm{He}$ also says:-_" The pea of our kitchen-gardens and fields is usually distinguished as a genus under the name of pisum, but upon characters which are hardly sufficient for the separation of a solitary species." At p. I27, speaking now of vetches, he says:- "The tendrils distinguish it from all our leguminous plants, except the following genus pea, from which it is absolutely separated, chiefly by the style." With Bentham, therefore, a lathyrus is a pea.

I A communication with specimens to the Lancashire Veterinary Medical Association, 30th September 1897. 